University of Wollongong

Research Online

Faculty of Informatics - Papers (Archive)

Faculty of Engineering and Information

Sciences

$1-1-2009$

\title{
Novel Cooperative Communication Schemes for Space-Time-Frequency Coded MB-OFDM UWB
}

\author{
Le Chung Tran \\ University of Wollongong, Ictran@uow.edu.au \\ Xiaojing Huang \\ CSIRO, huang@uow.edu.au \\ Alfred Mertins \\ University of Luebeck, Germany, mertins@uow.edu.au \\ Eryk Dutkiewicz \\ Macquarie University, eryk@uow.edu.au
}

Follow this and additional works at: https://ro.uow.edu.au/infopapers

Part of the Physical Sciences and Mathematics Commons

\section{Recommended Citation}

Tran, Le Chung; Huang, Xiaojing; Mertins, Alfred; and Dutkiewicz, Eryk: Novel Cooperative Communication Schemes for Space-Time-Frequency Coded MB-OFDM UWB 2009, 1-6.

https://ro.uow.edu.au/infopapers/786

Research Online is the open access institutional repository for the University of Wollongong. For further information contact the UOW Library: research-pubs@uow.edu.au 


\title{
Novel Cooperative Communication Schemes for Space-Time-Frequency Coded MB-OFDM UWB
}

\author{
Abstract \\ Cooperative communication has been intensively considered for general wireless and sensor networks. \\ However, it has been almost untouched in Space-Time-Frequency Coded Multi-band OFDM Ultra- \\ Wideband (STFC MB-OFDM UWB) systems. This paper thus proposes two cross-layer designs for \\ cooperative communication, namely Simple Cooperative Communication Scheme (SCCS) and Advanced \\ Cooperative Communication Scheme (ACCS), in slow fading scenarios. In the former, nodes always \\ cooperate with one another despite the possibly erroneous reception at the partner nodes, while in the \\ latter, nodes cooperate only in the case of successful reception. Simulations show that these schemes \\ may considerably improve the performance of STFC MB-OFDM UWB without any increase of \\ transmission power.

\section{Keywords} \\ space, uwb, schemes, ofdm, communication, cooperative, novel, mb, coded, frequency, time

\section{Disciplines} \\ Physical Sciences and Mathematics

\section{Publication Details} \\ Tran, L., Mertins, A., Huang, X. \& Dutkiewicz, E. (2009). Novel Cooperative Communication Schemes for \\ Space-Time-Frequency Coded MB-OFDM UWB. In T. A.. Wysocki (Eds.), 3rd International Conference on \\ Signal Processing and Communication Systems (pp. 1-6). Omaha, USA: IEEE.
}




\title{
Novel Cooperative Communication Schemes for Space-Time-Frequency Coded MB-OFDM UWB
}

\author{
L. C. Tran, A. Mertins, X. Huang, and E. Dutkiewicz
}

\begin{abstract}
Cooperative communication has been intensively considered for general wireless and sensor networks. However it has been almost untouched in Space-Time-Frequency Coded Multi-band OFDM Ultra-Wideband (STFC MB-OFDM UWB) systems. This paper thus proposes two cross-layer designs for cooperative communication, namely Simple Cooperative Communication Scheme (SCCS) and Advanced Cooperative Communication Scheme (ACCS), in slow fading scenarios. In the former, nodes always cooperate with one another despite the possibly erroneous reception at the partner nodes, while in the latter, nodes cooperate only in the case of successful reception. Simulations show that these schemes may considerably improve the performance of STFC MB-OFDM UWB without any increase of transmission power.
\end{abstract}

\section{INTRODUCTION}

Application of the emerging technologies, including Multiple Input Multiple Output (MIMO) [1], [2] and SpaceTime Codes (STCs) [3], [4], [5], to Multi-band OFDM UltraWideband (MB-OFDM UWB) [6] communications has recently attracted considerable attention in order to improve the performance and the capacity of MB-OFDM systems. Although the integration of normal OFDM, MIMO and STCs (referred to as STFC-MIMO-OFDM) has been intensively considered, the association between MB-OFDM UWB, MIMO and STCs [7], [8], has not been adequately examined yet .

Conventional OFDM systems and in MB-OFDM UWB ones differ in the following two aspects. First, channels in the latter are much more dispersive than those in the former, with the average number of multipaths in some channel models reaching some thousands [9]. Second, channel coefficients in the former are usually considered to be Rayleigh distributed, while those in the latter are log-normally distributed [9]. Therefore, the systems incorporating MB-OFDM UWB, MIMO, and STCs must be more specifically analyzed, though there exist several similarities between those systems and the conventional STFCMIMO-OFDM ones. In [10], [11], [12], [13], we proposed the framework of STFC MB-OFDM UWB systems for multiple number of transmit/reveive (Tx/Rx) antennas (see Fig. 1).

Two questions that could be raised are whether the principle of STFCs can be applied to a MB-OFDM UWB network where all source nodes (the transmitters, such as portable devices, which transmit the source information to the destination) are equipped with only one antenna due to their tiny physical size, while the destination node, such as a router, might be equipped with multiple Rx antennas, and in which scenario this application is useful. The answer for the first question is yes through the implementation of cooperative communication between nodes. The basic idea of cooperative communication is that single-antenna nodes can gain some of the benefits of MIMO systems by sharing their antennas with each other to create a virtual MIMO system.

Though cooperative communication has been intensively examined for general wireless networks with various exhaustive works, such as [14], [15], [16], [17], [18], [19], [20], it has been almost unexplored for MB-OFDM UWB. By extending further the discussions in the aforementioned works while taking the MB-OFDM UWB technical specifications [6] into account, in this paper, we propose for the first time two crosslayer designs for cooperative communication in STFC MBOFDM UWB, which are referred to as Simple Cooperative

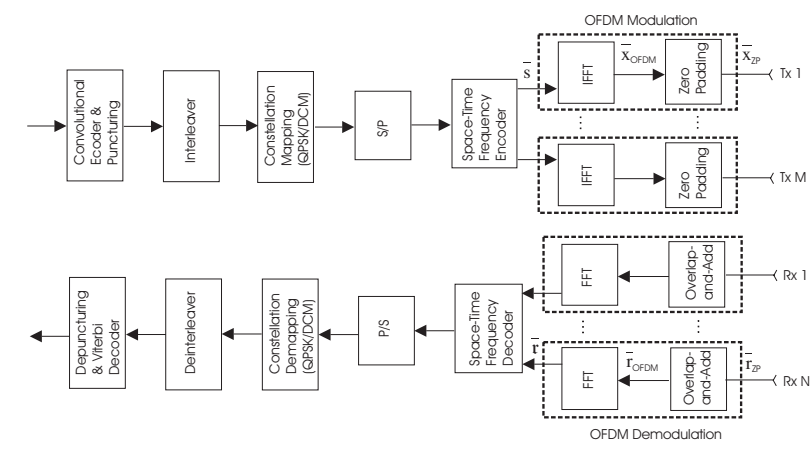

Fig. 1. Structural diagram of the proposed STFC MB-OFDM UWB system [10], [11], [12].

Communication Scheme (SCCS) and Advanced Cooperative Communication Scheme (ACCS), in slow fading scenario. The slow fading UWB channels, where channel coefficients keep constant during multiple MB-OFDM symbol time slots, are usually found in short range indoor UWB communication. We also consider the error performance of these cooperative communication models in several scenarios to verify in which scenario the application of cooperative communication is useful for STFC MB-OFDM UWB.

The paper is organized as follows. Sections II and III present the proposed SCCS and ACCS respectively. Simulation results are shown in Section IV and conclusions are withdrawn in Section V.

Notations: The following notations will be used throughout the paper. The superscripts $(.)^{*}$ and $(.)^{T}$ denote the complex conjugation and transposition operation, respectively. We denote $\overline{\mathbf{a}} \bullet \overline{\mathbf{b}}$ to be the element-wise (or Hadamard) product of the two vectors $\overline{\mathbf{a}}$ and $\overline{\mathbf{b}} . N_{D}$ and $N_{f f t}$ are the number of data subcarriers and the FFT/IFFT size, respectively (for MB-OFDM UWB communications [6], $N_{D}=100$ and $N_{f f t}=128$ ). Further, $\overline{\mathbf{a}}$. ^ 2 denotes the element-wise power- 2 operation of $\overline{\mathbf{a}}$. The complex space $\mathcal{C}$ of a symbol $s$ denotes all potential possibilities that the symbol $s$ can take, while the $N_{D^{-}}$ dimensional complex space $\mathcal{C}^{N_{D}}$ of a $N_{D}$-length vector $\overline{\mathbf{s}}$ denotes all potential possibilities that the vector $\overline{\mathbf{s}}$ can take. We define $\overline{1}$ as a column vector of length $N_{D}$, whose elements are all 1. We denote $\|.\|_{F}$ to be the Frobenius norm. Finally, we refer the time required to transmit a MB-OFDM symbol to as a $M B-O F D M$ symbol time slot and that required to transmit a symbol within STFCs to as a STFC symbol time slot, which is the same as the MB-OFDM symbol time slot if the STFC symbol consists of data for a single MB-OFDM symbol or is the multiple of MB-OFDM symbol time slots if it consists of data for multiple MB-OFDM symbols.

\section{Simple Cooperative Communication Scheme (SCCS)}

This scheme is perhaps the closest to the idea of the fixed Detect-and-Forward schemes in [15], [19]. However, it is a cross-layer design specifically proposed for MB-OFDM UWB. The proposed model is depicted in Fig. 2. For the brevity, 


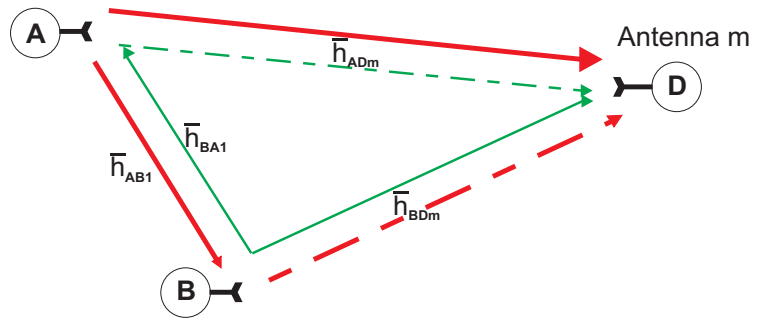

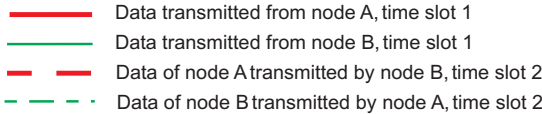

Fig. 2. Cooperative communication in MB-OFDM UWB between the source nodes $A, B$, and the $m$-th receive antenna of the destination node $D$ with the pairing mode.

our proposed STFC MB-OFDM UWB systems will not be reviewed in this paper. We would like to refer readers to [10, Section III] for more detail about how STFCs are constructed. We consider the application of the Alamouti STFC [3], [10]

$$
\mathbf{S}=\left[\begin{array}{rr}
\overline{\mathbf{s}}_{A_{i}} & \overline{\mathbf{s}}_{B_{i}} \\
-\overline{\mathbf{s}}_{B_{i}}^{*} & \overline{\mathbf{s}}_{A_{i}}^{*}
\end{array}\right]
$$

For the ease of explanation, we first consider the case where the STFC symbols $\overline{\mathbf{s}}_{A_{i}}$ and $\overline{\mathbf{s}}_{B_{i}}$ are the column vectors that consist of the original transmitted data (i.e. before the IFFT operation) and correspond to the $i$-th MB-OFDM symbols transmitted by the nodes $A$ and $B$, respectively. In fact, $\overline{\mathbf{s}}_{A_{i}}$ and $\overline{\mathbf{s}}_{B_{i}}$ are not necessarily restricted to be single MBOFDM symbols. Therefore, we will then consider the more generalized case where $\overline{\mathbf{s}}_{A_{i}}$ and $\overline{\mathbf{s}}_{B_{i}}$ are the packets of data corresponding to multiple MB-OFDM symbols.

It is assumed that nodes are perfectly synchronized. Denote $\overline{\mathbf{h}}_{j k m}=\left[\begin{array}{ll}h_{j k m, 1} & h_{j k m, 2} \ldots h_{j k m, L_{j k m}}\end{array}\right]^{T}$ to be the channel vector between the two nodes $j$ and $k$, at the $m$-th antenna of the node $m$, where $j \in\{A, B\} ; k \in\{A, B, D\},(k \neq j)$, and $m \in\{1,2, \ldots, N\}$ (see Fig. 2), while $L_{j \underline{k} m}$ is the number of multipaths in this link. The elements of $\overline{\mathbf{h}}_{j k m}$ are modeled as independent log-normally distributed random variables [9]. Channels are assumed to be slow fading ones, i.e. channel vectors $\overline{\mathbf{h}}_{j k m}$ are assumed to keep constant during several MBOFDM symbol time slots (MB-OFDM symbol time slots and STFC symbol time slots are the same in this case). The channel coefficients are assumed to be known at the destination node. Each of the source nodes $A$ and $B$ is equipped with only one antenna for transmitting and receiving signals, while the destination node $D$ might have $N$ antennas. In cooperative communication, each node transmits its own data as well as performs as a cooperative agent for another node.

In our proposed SCCS, two nodes are paired to cooperate with one another. The issue of how to decide which nodes to be paired with each other is out of scope of this paper. We assume that nodes $A$ and $B$ need to transmit the same number of MB-OFDM symbols (or in general, same numbers of packets). At the first MB-OFDM symbol time slot, node $A$ broadcasts its symbol $\overline{\mathbf{s}}_{A_{i}}$ to its partner (node $B$ ) and the destination node $D$. At the same time, node $B$ broadcasts its symbol $\overline{\mathbf{s}}_{B_{i}}$ to its partner (node $A$ ) and the destination node $D$. After receiving their partner's symbol, nodes $A$ and $B$ decode the partner's symbol. We denote the decoded symbols at nodes $A$ and $B$ to be $\overline{\mathbf{s}}_{B_{i}}$ and $\overline{\mathbf{s}}_{A_{i}}$ respectively. Then these two nodes retransmit the symbols to the destination in the form of $-\overline{\mathbf{s}}_{A_{i}}^{*}$ and $\overline{\mathbf{s}}_{B_{i}}^{*}$, respectively, during the second MB-OFDM symbol time slot. The process continues until all

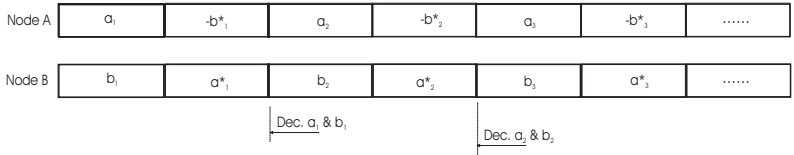

Fig. 3. Transmission protocol in the SCCS.

data are transmitted. This proposed scheme is thus referred to as decode-and-forward scheme. This scheme is simpler than some of the existing cooperative communication schemes, such as [15], [19], [20], with the penalty of loosing the flexible cooperation level between two nodes. The simplicity of the proposed scheme results in a low system cost which is one of the main requirements for UWB systems. The transmission protocol in the SCCS is depicted in Fig. 3. The decoding process of the $i$-th couple of MB-OFDM symbols $a_{i}$ and $b_{i}$ at the destination node starts after $2 i$ MB-OFDM symbol time slots.

After the overlap-and-add operation (OAAO) [6], [10] and FFT have been performed, the signals received at the $m$ antenna of the destination node $D$ during the two MB-OFDM symbol time slots can be represented as

$$
\begin{aligned}
& \overline{\mathfrak{r}}_{1 m}=\overline{\mathfrak{h}}_{A D m} \bullet \overline{\mathbf{s}}_{A_{i}}+\overline{\mathfrak{h}}_{B D m} \bullet \overline{\mathbf{s}}_{B_{i}}+\overline{\mathfrak{n}}_{1 m} \\
& \overline{\mathfrak{r}}_{2 m}=-\overline{\mathfrak{h}}_{A D m} \bullet \overline{\mathbf{s}}_{B_{i}}^{*}+\overline{\mathfrak{h}}_{B D m} \bullet \overline{\mathbf{s}}_{A_{i}}^{*}+\overline{\mathfrak{n}}_{2 m}
\end{aligned}
$$

where $\overline{\mathfrak{h}}_{j k m}:=\operatorname{FFT}\left(\overline{\mathbf{h}}_{j k m}\right), \overline{\mathfrak{n}}_{t m}:=\operatorname{FFT}\left(\overline{\mathbf{n}}_{t m}\right)$, while

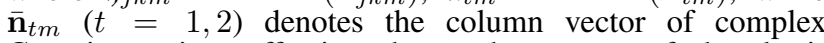
Gaussian noises affecting the $m$-th antenna of the destination node at the $t$-th MB-OFDM symbol time slot. Denote $\overline{\mathfrak{h}}_{j k m}=\left[\begin{array}{ll}\hbar_{j k m, 1} & \hbar_{j k m, 2} \ldots \hbar_{j k m, N_{f f t}}\end{array}\right]^{T}$ and $\overline{\mathfrak{r}}_{t m}=$ $\left[\mathfrak{r}_{t m, 1} \mathfrak{r}_{t m, 2} \ldots \mathfrak{r}_{t m, N_{f f t}}\right]^{T}$.

Once the destination node receives the symbols transmitted during the two MB-OFDM symbol time slots, it is able to decode the symbols. In particular, if we assume theoretically that the information transmitted from the source nodes is error-freely received at their partner, i.e. $\dot{s}_{A_{i}} \equiv \overline{\mathbf{s}}_{A_{i}}$ and $\overline{\mathbf{s}}_{B_{i}} \equiv \overline{\mathbf{s}}_{B_{i}}$, the detailed maximum likelihood (ML) decoding metrics in the cases of PSK or QAM modulation schemes can be found in Table I (see also [10, Table II]). Clearly, from Table I, instead of having to decode jointly the whole $N_{D}$ data points corresponding to the $N_{D}$ data sub-carriers $\left(N_{D}=100\right.$ according to [6]) within each of the MB-OFDM symbols $\overline{\mathbf{s}}_{A_{i}}$ and $\overline{\mathbf{s}}_{B_{i}}$, each data point among these $N_{D}$ data sub-carriers can be decoded separately. Thus the decoding process is completely linear, and relatively simple. Particularly, the decoding metrics for data at the $n$-th sub-carrier, for $n=1, \ldots, N_{D}$, in the MB-OFDM symbols $\overline{\mathbf{s}}_{A_{i}}$ and $\overline{\mathbf{s}}_{B_{i}}$ are

$$
\begin{aligned}
s_{A_{i}, n}= & \arg \min _{s \in \mathcal{C}}\left\{\left|\sum_{m=1}^{N}\left(\hbar_{A D m, n}^{*} \mathfrak{r}_{1 m, n}+\hbar_{B D m, n} \mathfrak{r}_{2 m, n}^{*}\right)-s\right|^{2}\right. \\
& \left.+\left[-1+\sum_{m=1}^{N}\left(\left|\hbar_{A D m, n}\right|^{2}+\left|\hbar_{B D m, n}\right|^{2}\right)\right]|s|^{2}\right\} \\
s_{B_{i}, n}= & \arg \min _{s \in \mathcal{C}}\left\{\left|\sum_{m=1}^{N}\left(\hbar_{B D m, n}^{*} \mathfrak{r}_{1 m, n}-\hbar_{A D m, n} \mathfrak{r}_{2 m, n}^{*}\right)-s\right|^{2}\right. \\
& \left.+\left[-1+\sum_{m=1}^{N}\left(\left|\hbar_{A D m, n}\right|^{2}+\left|\hbar_{B D m, n}\right|^{2}\right)\right]|s|^{2}\right\} .
\end{aligned}
$$

As mentioned previously, $\overline{\mathbf{s}}_{A_{i}}$ and $\overline{\mathbf{s}}_{B_{i}}$ in (1) are not necessarily the column vectors corresponding to single MB-OFDM symbols. In the more generalized scenario, they may be the $i$-th packets transmitted from nodes $A$ and $B$ that correspond to multiple MB-OFDM symbols. In practice, $\overline{\mathbf{s}}_{A_{i}}$ and $\overline{\mathbf{s}}_{B_{i}}$ can even be the column vectors of original transmitted data (before the IFFT) of multiple MB-OFDM symbols which are then transformed through the symbol-wise IFFT operation and 
TABLE I

DeCODING METRICS IN PSK OR QAM MOdulations.

\begin{tabular}{|c|c|}
\hline Symbol & Decoding Metric \\
\hline$\overline{\mathbf{s}}_{A_{i}}$ & $\begin{array}{l}\arg \min _{\overline{\mathbf{s}} \in \mathcal{C}^{N} D} \|\left\{\mid \sum_{m=1}^{N}\left(\overline{\mathfrak{h}}_{A D m}^{*} \bullet \overline{\mathfrak{r}}_{1 m}+\overline{\mathfrak{h}}_{B D m} \bullet \overline{\mathfrak{r}}_{2 m}^{*}\right)\right. \\
-\overline{\mathbf{s}} \mid \cdot{ }^{\wedge} 2+\left[-\overline{\mathbf{1}}+\sum_{m=1}^{N}\left(\left|\overline{\mathfrak{h}}_{A D m}\right| .^{\wedge} 2+\left|\overline{\mathfrak{h}}_{B D m}\right| .^{\wedge} 2\right)\right] \\
\left.\bullet\left(|\overline{\mathbf{s}}| \cdot^{\wedge} 2\right)\right\} \|_{F}^{2}\end{array}$ \\
\hline$\overline{\mathbf{s}}_{B_{i}}$ & $\begin{array}{l}\arg \min _{\overline{\mathbf{s} \in \mathcal{C}^{N}}} \|\left\{\mid \sum_{m=1}^{N}\left(\overline{\mathfrak{h}}_{B D m}^{*} \bullet \overline{\mathfrak{r}}_{1 m}-\overline{\mathfrak{h}}_{A D m} \bullet \overline{\mathfrak{r}}_{2 m}^{*}\right)\right. \\
-\overline{\mathbf{s}} \mid \cdot^{\wedge} 2+\left[-\overline{\mathbf{1}}+\sum_{m=1}^{N}\left(\left|\overline{\mathfrak{h}}_{A D m}\right| .^{\wedge} 2+\left|\overline{\mathfrak{h}}_{B D m}\right| \cdot^{\wedge} 2\right)\right] \\
\left.\bullet\left(|\overline{\mathbf{s}}| \cdot^{\wedge} 2\right)\right\} \|_{F}^{2}\end{array}$ \\
\hline
\end{tabular}

concatenated to form the whole payload of the Physical layer convergence protocol Service Data Units (PSDUs) transmitted from $A$ and $B$, respectively. The proposed ACCS is belong to this case which will be mentioned in more detail later in the next section.

Mathematically, we can consider them as column vectors which stack data corresponding to multiple MB-OFDM symbols on each other. The column vectors $\mathfrak{h}_{j k m}, \overline{\mathfrak{n}}_{t m}$ and $\overline{\mathfrak{r}}_{t m}$ in (2) are similarly constructed. The decoding is carried out at the receiver in a symbol-wise manner and the decoding metric for each MB-OFDM symbol within the packet $\overline{\mathbf{s}}_{A_{i}}$ or $\overline{\mathbf{s}}_{B_{i}}$ is detailed in Table I. Note that, instead of having to wait until the whole payloads of PSDUs have been received, the decoding process can be started as soon as the receiver receives signals during the first two MB-OFDM symbol time slots (the Alamouti STFC is being considered). Hence, decoding delay in the more generalized case has no difference, compared to that in the case where $\overline{\mathbf{s}}_{A_{i}}$ and $\overline{\mathbf{s}}_{B_{i}}$ represent single MB-OFDM symbols as mentioned above.

To solve the problem of transmission and reception at the same time at a node, in the existing works [19], [20], a code division multiple access (CDMA) was proposed. This means that each node is assigned with an unique spreading code, thus the two nodes can work in the same band. Unlike the existing proposed schemes, we take advantage of the important technical specifications of MB-OFDM UWB devices that, support for the first band group (3168 - $4752 \mathrm{MHz}$, see [6, Tables 7-1 \& 7-7]) is mandatory, and that the Time Frequency Code (TFCs) numbers 5, 6 and 7 for the first band group are non-overlapped with each other (see [6, Table 7-2]). Thus, in order for the nodes to be able to transmit their own data and receive the partner's data at the same time via only one antenna, node $A$ may, for instance, transmit signals by using TFC 5 (i.e. the radio frequency (RF) is in the range 3168 $3696 \mathrm{MHz}$ corresponding to the band number 1) and receive signals by using TFC 6 (i.e. RF in the range $3696-4224$ $\mathrm{MHz}$, band number 2). On the contrary, node $B$ may transmit signals by using TFC 6 and receive signals by using TFC 5 . The destination node must be able to work with both band numbers 1 and 2. This example is shown in Fig. 4. The principle of transmitting information in one frequency band and receiving information in another frequency band has been widely implemented, such as at the transponders in satellite communications. A node informs other nodes about its TFC by broadcasting its TFC in the 3-bit TX_TFC field (bits $T_{1}-T_{3}$ ) within the PHY (Physical Layer) header [6, p.28].

Since the inherent design of MB-OFDM UWB devices might have already allowed them to work with different TFCs (i.e. different bands) in the first band group, the only further tasks are to make source nodes to be able to transmit signals in one band, and receive signals in another band simultaneously, while making the destination node to be able to receive signals

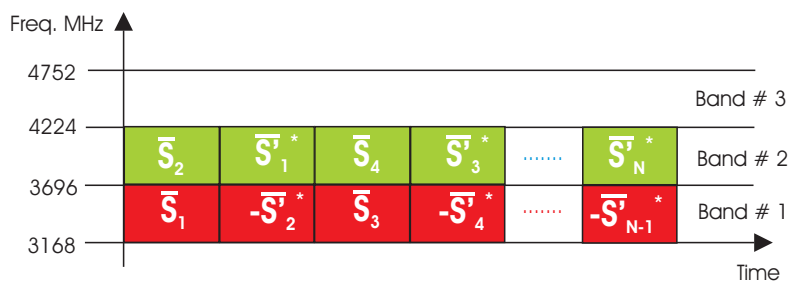

Fig. 4. Node $A$ transmits symbols in the band number 1 , while node $B$ transmits symbols in the band number 2 , inside the first band group.

from two different bands at the same time. These are not hassling tasks thanks to the implementation of precise filters. As a result, the design of transmitter/receiver at nodes can be created by modifying their current design without additional heavy complexity.

Clearly, in the SCCS, nodes always cooperate with one another regardless of the potentially erroneous reception of data from their partner. Thus the advantage of the SCCS is that it may provide a significant improvement of the system error performance in some cases (this will be confirmed later through simulation results) while keeping the nodes' design simple and as a result at a low cost. The disadvantage of the SCCS is that errors in decoding processes at the partner nodes may exit, i.e. $\overline{\mathbf{s}}_{A_{i}} \neq \overline{\mathbf{s}}_{A_{i}}$ and $\dot{\mathbf{s}}_{B_{i}} \neq \overline{\mathbf{s}}_{B_{i}}$, thus decoding errors at the destination node are the accumulative errors of the decoding processes at the partner nodes as well as the decoding process at the destination node. Intuitively, when the errors at the partner nodes become serious, the advantage of higher transmission diversity gained by the cooperation between nodes over non-cooperative communication can be ruined. As a result, SCCS might not be better than noncooperative communication in this case. Thus proposition of an advanced scheme that can provide better error performance than non-cooperative communication in all cases with the penalty of minimum additional complexity and cost is our next motivation. The advanced scheme referred to as ACCS will be detailed in the next section.

\section{Advanced Cooperative Communication Scheme (ACCS)}

This scheme is perhaps the closest to the ideas of the hybrid Detect-and-Forward schemes in [15] and of the space-time coded cooperative communication in [21]. However, it is a cross-layer design specifically proposed for MB-OFDM UWB and has various different aspects, compared to these existing schemes. The core idea of the proposed ACCS is that two source nodes $A$ and $B$ will decide whether the Alamouti STFC cooperative mode or non-cooperative mode will be effective, depending on whether they both receive correctly data from their partner. It should be noted that, though the data of a source node might be correctly received at its partner, this does not necessarily mean that the destination node also can receive correctly the data. This is because the distance between the cooperative nodes is normally shorter than that between these nodes and the destination.

In the ACCS, $\overline{\mathbf{s}}_{A_{i}}$ and $\overline{\mathbf{s}}_{B_{i}}$ are the column vectors consisting of the original data for the whole payload of PSDUs. Denote $p$ to be the current STFC symbol time slot. During the next time slot $p+1$, node $A$ (node $B$ respectively) broadcasts its own next packet $\overline{\mathbf{s}}_{A_{i+1}}\left(\overline{\mathbf{s}}_{B_{i+1}}\right)$ together with the acknowledge bit $A C K_{A_{i}}\left(A C K_{B_{i}}\right)$. The value of the acknowledge bits depend on whether node $A$ (B) receives correctly the $i$-th packet $\overline{\mathbf{s}}_{B_{i}}$ from $B\left(\overline{\mathbf{s}}_{A_{i}}\right.$ from $\left.A\right)$ during the time slot $p$. In particular, the acknowledge bit will be set to one if the data are correctly received by the partner node, and will be zero otherwise. Each node then checks the ACK bit received from its partner. If 


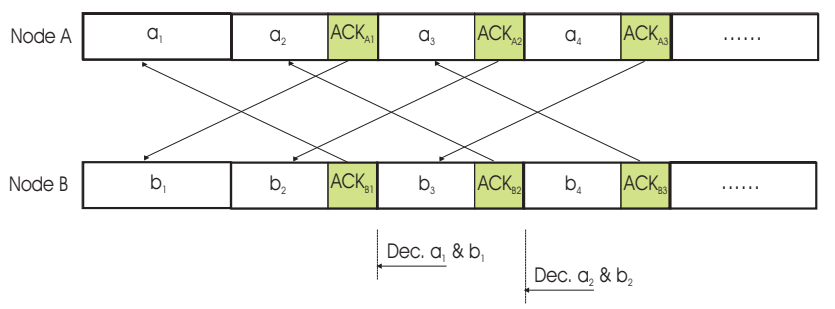

Case a: $\mathrm{ACK}_{\mathrm{A} 1}, \mathrm{ACK}_{\mathrm{B} 1}, \mathrm{ACK}_{\mathrm{A} 2}, \mathrm{ACK}_{\mathrm{B} 2}$ are all zeros.

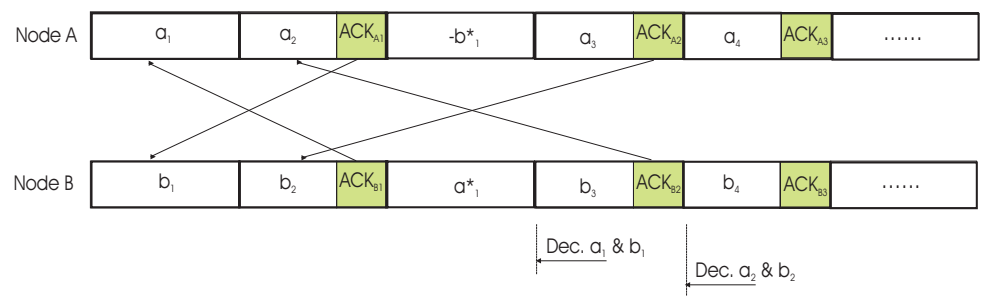

Case b: $\mathrm{ACK}_{\mathrm{A} 1}, \mathrm{ACK}_{\mathrm{B} 1}$ are ones while $\mathrm{ACK}_{\mathrm{A} 2}$ or $\mathrm{ACK}_{\mathrm{B} 2}$ or both of them are zeros.

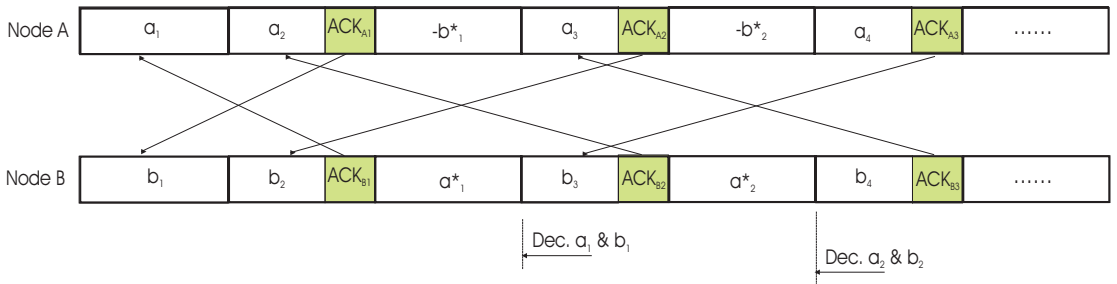

Case c: $\mathrm{ACK}_{\mathrm{A} 1}, \mathrm{ACK}_{\mathrm{B} 1}, \mathrm{ACK}_{\mathrm{A} 2}, \mathrm{ACK}_{\mathrm{B} 2}$ are all ones.

Fig. 5. Transmission protocol in the ACCS.

this bit and its own $\mathrm{ACK}$ bit $\left(A C K_{A_{i}}\right.$ and $\left.A C K_{B_{i}}\right)$ are all one, i.e. both nodes receive correctly data from their partner during the time slot $p$, then the Alamouti STFC cooperative mode will be formed. This means that, during the time slot $p+2$, node $A$ (B) halts broadcasting its $(i+2)$-th packet and broadcast its partner's data in the form of $-\overline{\mathbf{s}}_{B_{i}}^{*}\left(\overline{\mathbf{s}}_{A_{i}}^{*}\right)$ instead. Then nodes $A$ and $B$ continue to broadcast the $(i+2)$-th packet together with the ACK bits $A C K_{A_{i+1}}$ and $A C K_{B_{i+1}}$ during the time slot $p+3$. However, if at least one of the ACK bits $A C K_{A_{i}}$ and $A C K_{B_{i}}$ is zero, the non-cooperative mode will be used, i.e. nodes $A$ and $B$ continue to broadcast their $(i+2)$-th packets together with the ACK bits for the $(i+1)$-th packets during the time slot $p+2$. This process is repeated until all data are transmitted. It is noted that the ACCS does not use more STFC symbol time slots, compared to the SCCS, for data transmission. Instead, it requires at least the same number of time slots as non-cooperative communication (if nodes cannot receive correctly data from their partner at all), while requiring at most the same number of time slots as the SCCS (if otherwise), to transmit the same amount of data.

One may have a question: how can nodes check whether they receive correctly data from their partner? In the MBOFDM UWB design [6], the FCS (Frame Check Sequence) field of a PSDU is a 32-bit CRC (Cyclic Redundancy Check) sequence used by the MAC (Media Access Control) layer to check if the payload within this PSDU is correctly received.
Because STFC symbols $\overline{\mathbf{s}}_{A_{i}}$ and $\overline{\mathbf{s}}_{B_{i}}$ in our ACCS are the column vectors consisting of the original data for the whole payload of PSDUs, we take advantage of the FCS field to allow nodes to check if they receive correctly data from their partner. Thus we do not have to transmit any additional CRC bit for this purpose. By checking the field $F C S_{i}$ within the $i$ th PSDU, nodes can recognize whether they receive correctly the $i$-th STFC symbol within this PSDU.

At the destination node, decoding $i$-th packets that have been received from $A$ and $B$ during the time slot $p$ will be delayed by one STFC symbol time slot, i.e. decoding is carried out during the time slot $(p+2)$, if non-cooperative mode is the case, and by two STFC symbol time slots, i.e. decoding is carried out during the time slot $(p+3)$, if Alamouti cooperative mode is the case. During the time slot $(p+1)$, node $D$ continues to receive the $(i+1)$-th packets as well as the ACK bits $A C K_{A_{i}}$ and $A C K_{B_{i}}$ from $A$ and $B$. If both ACK bits are one, it will recognize that the Alamouti STFC cooperative mode is effective. Otherwise, the non-cooperative mode is the effective mode. In the case of the Alamouti STFC cooperative mode, the destination node receives the copies of the $i$-th packets in the form of $-\overline{\mathbf{s}}_{B_{i}}^{*}$ and $\overline{\mathbf{s}}_{A_{i}}^{*}$ from $A$ and $B$ during the time slot $(p+2)$, and then decodes them. In the case of non-cooperative mode, it can start decoding the $i$-th packets $\overline{\mathbf{s}}_{A_{i}}$ and $\overline{\mathbf{s}}_{B_{i}}$ in the time slot $(p+2)$. This process is repeated until all packets are received. 
Clearly, the aforementioned scheme will work precisely if and only if nodes receive correctly the values of ACK bits. Thus maximizing the chance of successful reception of ACK bits at nodes is our main target. To do that, we take advantage of the reserved bit fields $R_{0}-R_{2}, R_{20}-R_{21}, R_{24}-R_{25}$ and $R_{32}-R_{39}$ in the PHY header within the PLCP header of a PPDU (PLCP Protocol Data Unit) [6, p.28]. In particular, the acknowledge bit $A C K_{i}$ is transmitted in multiple positions among these 15 reserved bits, if not all. At the receiving side, a majority rule will be applied to realize the actual value of $A C K_{i}$. For instance, the acknowledge bit $A C K_{A_{i}}$ can be simultaneously transmitted in the three following positions $R_{0}$, $R_{20}$ and $R_{39}$ in the PHY header of the $(i+1)$-th PPDU. Nodes $B$ and $D$ can realize the actual value of $A C K_{A_{i}}(0$ or 1) by selecting the value that turns up more times than the other value among the above three positions. Certainly, the more positions we use for transmitting $A C K_{i}$, the more precisely the ACCS works. Other important inherent features of PPDUs include that the validity of the combined PHY and MAC headers of the PPDU will be checked by MAC using the HCS (Header Check Sequence) which is a 16-bit CRC code, and that the validity of the MAC header and HCS is one more time checked by the 48-bit Reed-Solomon parity bits. In addition, we note that because $F C S_{i}$ is used for checking the whole payload which may consist of up to 4095 octets, a single error in the payload can cause a false $F C S_{i}$ checking result. Thus even in the case where a node cannot receive correctly data from its partner (i.e. checking $F C S_{i}$ gives a false result), this does not necessarily mean that the node also cannot receive correctly the ACK bit, which is transmitted at several positions in the PHY header from its partner. It is also noted that the PHY header is always transmitted at the data rate of $39.4 \mathrm{Mbps}$ that is much lower than the transmission rate of the payload [6, p.9], [22, p.1180]. For all aforementioned analysis, it may be reasonable to assume that the ACK bits are correctly received at nodes in slow fading channels.

The following examples consider typical cases when the first two packets $a_{1}, a_{2}$ are transmitted from node $A$ and $b_{1}$, $b_{2}$ are transmitted from node $B$.

Example 1: This example is depicted in Case a, Fig. 5, where all ACK bits $A C K_{A_{1}}, A C K_{A_{2}}, A C K_{B_{1}}$ and $A C K_{B_{2}}$ are zeros. In practice, when an ACK bit is zero, it might not need to be transmitted. Intuitively, this case is similar to the non-cooperative communication, except for that the decoding process for the packets $a_{1}, b_{1}$ (and $a_{2}, b_{2}$ ) at the destination node starts at one STFC symbol time slot later than that in the non-cooperative communication.

Example 2: This example considers the case where the ACK bits $A C K_{A_{1}}$ and $A C K_{B_{1}}$ are ones while either $A C K_{A_{2}}$ or $A C K_{B_{2}}$ or both of them are zeros (see Case b, Fig. 5). In this case, the decoding process for the packets $a_{1}, b_{1}$ (and $a_{2}$, $b_{2}$ ) at the destination node is delayed by further one STFC symbol time slot compared to Example 1.

Example 3: Case $\mathrm{c}$ in Fig. 5 depicts the example where all ACK bits $A C K_{A_{1}}, A C K_{A_{2}}, A C K_{B_{1}}$ and $A C K_{B_{2}}$ are ones. The decoding process in this case is similar to that in the SCCS in Fig. 3, except for that the decoding process for packets starts at one STFC symbol time slot later than that in the SCCS.

From Examples 1-3, we can see that the starting point of decoding process in the ACCS is delayed by one STFC symbol time slot, compared to the SCCS, and the decoding process of an $i$-th packets is delayed at most $2(i-1)$ STFC symbol time slots, compared to this starting point. This is because the destination node has to wait for one more STFC symbol time slot before the decoding process for the $i$-th packets can start in the case the non-cooperative mode is effective, or has to wait for two more STFC symbol time slots if the Alamouti STFC cooperative mode is effective. Thus the advantages of the ACCS over the SCCS come with the cost of a decoding
TABLE II

SIMULATION PARAMETERS.

\begin{tabular}{l|r}
\hline Parameter & Value \\
\hline FFT and IFFT size & $N_{f f t}=128$ \\
Data rate & $320 \mathrm{Mbps}$ \\
Convolutional encoder's rate & $1 / 2$ \\
Convolutional encoder's constraint length & $K=7$ \\
Convolutional decoder & Viterbi \\
Decoding mode & Hard \\
STFC decoding at nodes & ML decoding \\
Number of transmitted packets & 1200 \\
Modulation & QPSK \\
IEEE Channel model & CM1, 2, 344 \\
Number of data subcarriers & $N_{D}=100$ \\
Number of pilot subcarriers & $N_{P}=12$ \\
Number of guard subcarriers & $N_{G}=10$ \\
Total number of subcarriers used & $N_{T}=122$ \\
Number of samples in ZPS & $N_{Z P S}=37$ \\
Total number of samples/symbol & $N_{S Y M}=165$ \\
Number of channel realizations & 100 \\
\hline \multicolumn{2}{|c}{} \\
\hline
\end{tabular}

delay of at most two STFC symbol time slots.

\section{Simulation Results}

To examine the performance advantage of the cooperative communication schemes, we ran several Monte-Carlo simulations for non-cooperative communication and for the SCCS and the ACCS. Each run of simulations was carried out with 1200 MB-OFDM symbols. One hundred channel realizations of each channel model (CM 1 to CM 4) were considered for the transmission of each MB-OFDM symbol. In simulations, $S N R$ is defined to be the signal-to-noise ratio $(\mathrm{dB})$ per sample in a MB-OFDM symbol (consisting of 165 samples), at each Rx antenna (i.e. the subtraction between the total power (dB) of the received signal corresponding to the sample of interest and the power of noise $(\mathrm{dB})$ at that $\mathrm{Rx}$ antenna). The complete set of simulation parameters is presented in Table II.

To fairly compare the error performance of non-cooperative and the two cooperative communication schemes, we stick to the following constraints

Data rate constraint: Simulations for all three systems are run with the same bit rate of 320 Mbps.

Power constraint: The average power of the signal constellation points is always scaled down by a factor of 2 in the SCCS, while it is scaled down by a factor of 2 whenever the Alamouti STFC mode is effective in the ACCS. Thereby, the total transmission power from the source nodes to the destination node at a certain time is the same for all three systems.

Fig. 6 compares the error performances of non-cooperative communication and of the SCCS in the case nodes are equipped with one antenna each. From Fig. 6, we can realize that the SCCS may provide better error performances than non-cooperative communication for the $S N R$ being greater than certain values $S N R_{\text {min }}$, which are 7,8 , and $10 \mathrm{~dB}$ in CM $1, \mathrm{CM} 2$ and CM 3, respectively. For instance, the SCCS brings about the gain of approximate $1.5 \mathrm{~dB}$ at $B E R=10^{-3}$, and approximate $3 \mathrm{~dB}$ at $B E R=4 \times 10^{-4}$, over non-cooperative communication in CM 1. Note that this advance is achieved when every node (including the destination node) is installed with one antenna and there is not any increase of transmission power. The error performance advantage is gained due to the fact that the diversity of the transmitted signals has been increased by the cooperation between nodes. It can also be realized from Fig. 6 that the use of the SCCS might not be useful for the very dispersive channel model CM 4.

The error performances of non-cooperative communication and of the ACCS in the case nodes are equipped with one antenna each are compared in Fig. 7. As opposed to the SCCS, the ACCS provides at least the same error performance as noncooperative communication at very low $S N R \mathrm{~s}$, while always providing better error performance than the non-cooperative 


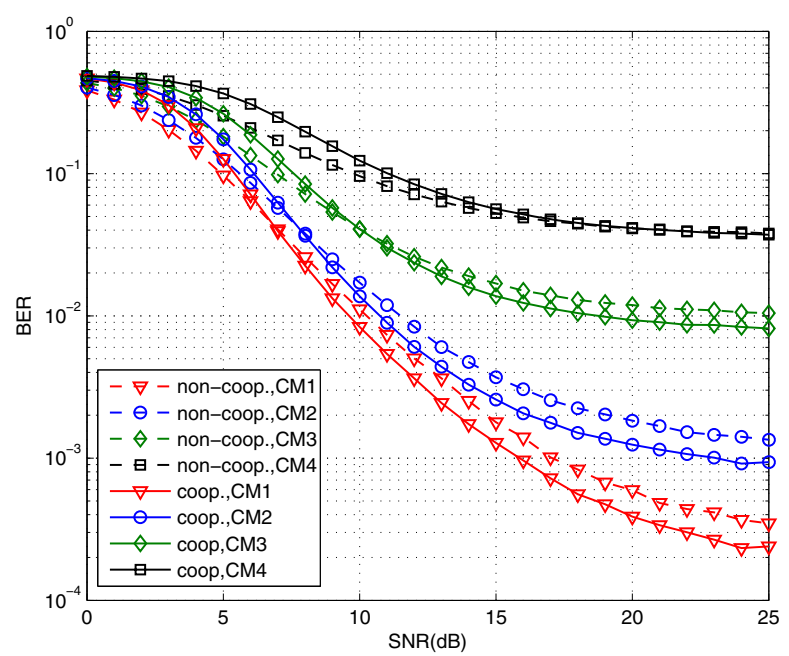

Fig. 6. SCCS vs. non-cooperative communication in MB-OFDM UWB with the one-antenna destination node.

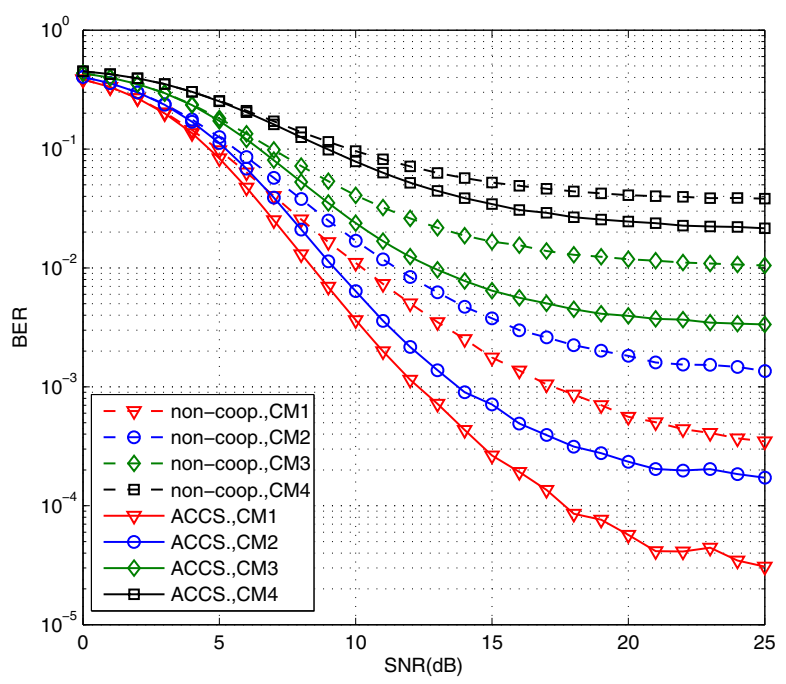

Fig. 7. ACCS vs. non-cooperative communication in MB-OFDM UWB with ACCA and with the one-antenna destination node.

communication at higher $S N R$ s in all channel models. For instance, the ACCS brings about the gain of approximate 5 $\mathrm{dB}$ at $B E R=10^{-3}$ over non-cooperative communication in CM 1. The more dispersive the channel is, the higher the gain is.

\section{CONCLUSIONS}

This paper has proposed two cross-layer designs, referred to as the SCCS and ACCS, for cooperative communication in STFC MB-OFDM UWB systems. From the simulation results, we might conclude that the benefit of the SCCS over non-cooperative communication is considerable in the network with all single-antenna nodes and in less dispersive UWB channel models, such as CM 1, 2 or 3 . In contrast, the benefit of the ACCS is significant in all cases with the penalties of the decoding delay at the destination, and a slightly more complicated communication protocol between nodes, compared to the SCCS, though both schemes are proposed in the sense that the additional complexity and costs are kept as little as possible. The performance improvements are gained without increase of total transmission power and without significant additional system complexity and cost.

\section{ACKNOWLEDGMENT}

L. C. Tran would like to thank the Alexander von Humboldt $(\mathrm{AvH})$ Foundation, Germany, for its support of this work under the postdoctoral research fellowship.

\section{REFERENCES}

[1] I. E. Telatar, "Capacity of multi-antenna Gaussian channels," European Trans. Telecom., vol. 10, no. 6, pp. 585-595, 1999.

[2] G. J. Foschini and M. J. Gans, On limits of wireless communications in a fading environment when using multiple antennas, vol. 6 of 3, Wireless Personal Communications, Printed in the Netherlands, Mar. 1998.

[3] S. M. Alamouti, "A simple transmit diversity technique for wireless communications," IEEE J. Select. Areas Commun., vol. 16, no. 8, pp. $1451-1458$, Oct. 1998

[4] V. Tarokh, N. Seshadri, and A. R. Calderbank, "Space-time codes for high data wireless communications: performance criterion and code construction," IEEE Trans. Inform. Theory, vol. 44, no. 2, pp. $744-$ 765, Mar. 1998

[5] L. C. Tran, T. A. Wysocki, A. Mertins, and J. Seberry, Complex Orthogonal Space-Time Processing in Wireless Communications, Springer, New York, USA, 2006.

[6] A. Batra et al., "Multiband OFDM physical layer specification.," WiMedia Alliance Release 1.1, July 2005.

[7] T.-H. Tan and K.-C. Lin, "Performance of space-time block coded MBOFDM UWB systems," Proc. 4th Annual Communication Networks and Services Research Conference (CNSR'06), pp. 323 - 327, May 2006.

[8] W. P. Siriwongpairat, W. Su, M. Olfat, and K. J. R. Liu, "MultibandOFDM MIMO coding framework for UWB communication systems," IEEE Trans. Sign. Process., vol. 54, no. 1, pp. $214-224$, Jan. 2006.

[9] J. Foerster and Intel R\&D, "Channel modelling sub-committee report final," IEEE P802.15 Working Group for Wireless Personal Area Networks (WPANS), IEEE P802.15-02/490r1-SG3a, Oct. 2005.

[10] L. C. Tran and A. Mertins, "Space-time frequency code implementation in MB-OFDM UWB communications: design criteria and performance," IEEE Trans. Wireless Commun., vol. 8, no. 1, pp. 701-713, Feb. 2009

[11] L. C. Tran, A. Mertins, E. Dutkiewicz, and X. Huang, "Space-timefrequency codes in MB-OFDM UWB communications: Advanced order8 STFC and its performance," Proc. 7th IEEE International Symposium on Communications and Information Technologies ISCIT 2007, Oct. 2007.

[12] L. C. Tran, A. Mertins, and T. A. Wysocki, "Quasi-orthogonal spacetime-frequency codes in MB-OFDM UWB communications," Proc. International Conference on Signal Processing and Communication Systems ICSPCS2007, Dec. 2007.

[13] L. C. Tran and A. Mertins, "On the use of quasi-orthogonal space-timefrequency codes in MB-OFDM UWB," Proc. 2nd IEEE International Conference on Communication and Electronics (ICCE2008), June 2008.

[14] Todd E. Hunter and Aria Nosratinia, "Diversity through coded cooperation," IEEE Trans. Wireless Commun., vol. 5, no. 2, pp. 283-289, Feb. 2006.

[15] J. Nicholas Laneman, David N. C. Tse, and Gregory W. Wornell, "Cooperative diversity in wireless networks: efficient protocols and outage behavior," IEEE Trans. Inform. Theory, vol. 50, no. 12, pp. 3062-3080, Dec. 2004

[16] X. Li, "Energy efficient wireless sensor networks with transmission diversity," IEE Electronics Lett., vol. 39, no. 24, Nov. 2003.

[17] P. Mitran, H. Ochiai, and V. Tarokh, "Space-time diversity enhencements using collaborative communications," IEEE Trans. Inform. Theory, vol. 51 , no. 6 , pp. 2041-2057, June 2005

[18] A. Norsratinia, T. E. Hunter, and A. Hedayat, "Cooperative communication in wireless networks," IEEE Commun. Magazine, vol. 42, no. 10 pp. 74-80, Oct. 2004.

[19] Andrew Sendonaris, Elza Erkip, and Behnaam Aazhang, "User cooperation diversity - part I: system description," IEEE Trans. Commun., vol. 51, no. 11, pp. 1927-1938, Nov. 2003.

[20] Andrew Sendonaris, Elza Erkip, and Behnaam Aazhang, "User cooperation diversity - part II: implementation aspects and performance operation diversity - part II: implementation aspects and performa

[21] J. N. Laneman and G. W. Wornell, "Distributed spacetime-coded protocols for exploiting cooperative diversity in wireless networks," IEEE Trans. Inform. Theory, vol. 49, no. 10, pp. 2415-2425, Oct. 2003

[22] R. S. Sherratt, "Design issues toward a cost effective physical layer for multiband ofdm (ecma-368) in consumer products," IEEE Transaction on Consumer Electronics, vol. 52, no. 4, pp. 1179-1183, Nov. 2006. 